\title{
A Transient and Flexible Cation- $\pi$ Interaction Promotes Hydrolysis of Nucleic Acids in DNA and RNA Nucleases
}

Vito Genna, ${ }^{1}$ Marco Marcia, ${ }^{2}$ Marco De Vivo ${ }^{1,}$

${ }^{1}$ Laboratory of Molecular Modeling and Drug Discovery, Istituto Italiano di Tecnologia, Via Morego 30, 16163, Genoa, Italy

${ }^{2}$ European Molecular Biology Laboratory, Grenoble Outstation, 71 Avenue des Martyrs, Grenoble 38042, France

\section{Supporting Information}

* Corresponding author

Marco De Vivo

marco.devivo@iit.it 
FF-BASED MOLECULAR DYNAMICS.

CAR-PARRINELLO MOLECULAR DYNAMICS (QM/MM MD).

BORN-HOPPENHEIMER MOLECULAR DYNAMICS (QM/MM MD).

SEQUENCE AND STRUCTURE-BASED ALIGNMENTS

SUPPLEMENTARY FIGURE S1.

SUPPLEMENTARY FIGURE S2.

S7

SUPPLEMENTARY FIGURE S3.

58

SUPPLEMENTARY FIGURE S4.

SUPPLEMENTARY FIGURE S5.

S10

SUPPLEMENTARY FIGURE $\mathbf{S 6 .}$

S11

SUPPLEMENTARY FIGURE S7.

S12

SUPPLEMENTARY FIGURE S8.

S13

REFERENCES

S14 


\section{Supplementary Text}

FF-based Molecular Dynamics. Theoretical calculations were performed on three different systems: i) protonated Lys131, ii) deprotonated Lys131 and iii) Lys-to-Ala mutation. All of them were generated starting from two different $\mathrm{X}$-ray structures. The wild-type structure containing $\mathrm{Ca}^{2+}$ in the active site (i.e. non-reactive wild-type Exo- $\lambda /$ dsDNA binary complex, PDBid 4 WUS) ${ }^{1}$ and the Lys131Ala isoform containing the $\mathrm{Mg}^{2+}$ in the active site (PDBid 3SM4). ${ }^{2}$ After system modeling and ions replacement, the catalytic Michaelis-Menten complex containing both Lys131 and $\mathrm{Mg}^{2+}$ ions was generated. The all-atom AMBER/FF14SB ${ }^{3}$ and $\mathrm{AMBER} / \mathrm{parmbsc}^{4}$ force-field were used to describe the system. The length of all covalent bonds, including hydrogen atoms, was set using the LINCS algorithm, ${ }^{5}$ allowing a time-integration step of 2 fs. All simulations were performed using GROningen MAchine for Chemical Simulations (Gromacs) 2016 code. ${ }^{6}$ Long-range electrostatic interactions were calculated with the particle mesh Ewald method (PME) with a real space cut-off of $12 \AA$ and periodic boundary conditions in the three directions of Cartesian space were used. Constant temperature $(310 \mathrm{~K})$ was imposed using Langevin dynamics ${ }^{7}$ with a damping coefficient of 1 ps. A constant pressure of $1 \mathrm{~atm}$ was maintained with Langevin-Piston dynamics ${ }^{8}$ with a $200 \mathrm{fs}$ decay period and a 50 fs time constant. All the simulated systems were hydrated using TIP3P water molecules. ${ }^{9} \mathrm{Na}^{+}$and $\mathrm{Cl}^{-}$ions were added to neutralize the total charge of the system. The size of the final box was approximately $105 \AA \times 110 \AA \times 75 \AA$, with $\sim 21000$ water molecules, resulting in a total number of $\sim 75000$ atoms each. The simulation protocol we have adopted is the following: all the systems were initially minimized using a steepest-descent algorithm and then were slowly heated up to $310 \mathrm{~K}$ in $10 \mathrm{~ns}$. The first $50 \mathrm{~ns}$ of production run are considered as equilibration phase. Approximately $1.5 \mathrm{~ns}$ of MD simulations were collected in the NPT ensemble for each of the three systems, resulting in a total of $4.5 \mu$ s of dynamics. Coordinates of the systems were collected every 5 ps and statistics were collected considering the equilibrated trajectories only (thus discarding the first $\sim 50 \mathrm{~ns}$ of simulation for each systems). 
Car-Parrinello Molecular Dynamics (QM/MM MD). Car-Parrinello MD is an established ab initio method that does not rely on empirically determined potentials. ${ }^{10}$ Instead, internuclear forces are determined on-the-fly from electronic structure calculations using the Kohn-Sham formulation of the Density Functional Theory (DFT). ${ }^{11}$ Such approach, despite being much more computationally demanding than classical and semiempirical methods, has been demonstrated to be robust and informative when used to study chemical reactivity, including systems based on metals and their complexes. Due to its high computational cost, a full QM treatment of the whole system (about $\sim 130,000$ atoms) would not be possible. Thus, we rely on a hybrid QM/MM potential ${ }^{12}$ to investigate dsDNA hydrolysis performed by phage- $\lambda$ exonuclease. ${ }^{2}$ According to this approach, the QM description is reserved to the enzyme's active site (including the two catalytic metals and a relevant portion of the DNA substrate), while the remaining part of the system is treated at classical level. In detail, the reactive region of the complex - 137 atoms in total, including $\mathrm{MgA}$ and $\mathrm{MgB}$ and the K131, Y154, D119, E120, L130 residues, seven water molecules and key atoms of the nucleic acid involved in the reaction - was treated at the DFT level using the BLYP functional. ${ }^{13 ; 14}$ This level of theory has been shown to describe well a variety of enzymatic reactions, with a fairly good (semiqualitative) estimation of the energetics for catalysis. ${ }^{15-18}$

The remaining part of the system, including all the other water molecules and counterions, was treated at the AMBER14 force field with parmbsc1 corrections. ${ }^{3 ; 4}$ In more details, the wave functions are expanded in a plane wave basis set up to 75 Ry in a QM cell of proper dimensions, taking as reference our previous studies. ${ }^{15 ; 19 ; 20}$ Only the valence electrons are treated explicitly (in the case of $\mathrm{Mg}$ atoms, electrons in the $\mathrm{n}=5$ shell are also included in the valence), while the core electrons will be described using norm-conserving pseudopotentials of the Martins-Troullier type. ${ }^{21}$ An adapted monovalent carbon pseudopotential is employed to saturate the dangling bonds in between the QM and $\mathrm{MM}$ regions. Isolated system conditions ${ }^{22}$ is imposed in the QM part by employing the MartynaTuckerman scheme. ${ }^{23}$ Notably, this approach has been shown to accurately describe a variety of enzymatic systems and protein/DNA complexes. ${ }^{18 ; 24 ; 25}$ All simulations were performed with the code CPMD (www.cpmd.org) version 4.1.1, developed at IBM Research Division, Zürich and the MaxPlanck Institute, Stuttgart. This program features an efficient QM/MM interface developed by Prof. Röthlisberger and co-workers ${ }^{12}$ based on the Gromos MD engine. ${ }^{26}$ 
Born-Hoppenheimer Molecular Dynamics (QM/MM MD). BO MD, performed via AMBER program package patched with Terachem $1.9,{ }^{27-30}$ has been used to estimate the free energy surface associated to the deprotonation of Lys131 via either Asp138 or through a bulk water molecule in Exonuclease- $\lambda$ (Exo- $\lambda$ ). These were performed with a QM subsystem of 82 atoms for the Aspmediated Lys131deprotonation and 76 atoms for Wat-mediated one. The QM part was described at the BLYP/6-31 G* level of theory, an approach successfully and largely used to well reproduce phosphate reactivity. ${ }^{31 ; 32}$ The link method (as implemented in AMBER) was used to saturate the valence of those atoms separating the QM subsystem from the MM part. ${ }^{27,28}$ The hybrid QM/MM models were built by using randomly selected snapshots obtained in the last $50 \mathrm{~ns}$ of unbiased forcefield based MD simulations. These, were then minimized and re-equilibrated (50 ps long NPTensemble QM/MM-MD simulation) at the BLYP/6-31 G*/MM level of theory, ${ }^{33}$ from which a spherical droplet (maintaining at least $10 \mathrm{~A}^{\circ}$ of solvent around Exo- $\lambda / \mathrm{dsDNA}$ binary complex) was extracted to compute the 2D BLYP/6-31 PESs. One reaction coordinate was monitored to specifically controlling the proton-transfer $\left[\mathrm{RC}=\mathrm{r} 1-\mathrm{r} 2\right.$, where $\mathrm{r} 1=d \mathrm{~N}_{\text {Lys }}-\mathrm{H}_{\text {Lys }}$ (breaking bond) and $\mathrm{r} 2=\mathrm{H}_{\text {Lys }}-$ $\mathrm{O}_{\text {Wat }}$ (forming bond)].

Reconstruction of the free energy surface (FES) was performed by QM/MM-MD simulations and the umbrella sampling procedure was used to compute the potential of mean force (PMF) associated to the proton-transfer event. ${ }^{31-33}$ Once stable, PMF were subsequently integrated by means of WHAM method to reconstruct the QM/MM-MD FESs. ${ }^{34}$ Statistical uncertainties for the PMF were calculated making use of the Bayesian bootstrap technique. ${ }^{35}$ The 2D PMF was obtained using PES structures derived above from QM/MM calculations as starting points. FESs were reconstructed from 10 simulation windows, each consisting of $10 \mathrm{ps}$ of equilibration and $30 \mathrm{ps}$ of well-equilibrated trajectory. Thus, a total simulation time of 400 ps at the BLYP/6-31 G*/MM level of theory were performed. Electrostatic embedding was used in all hybrid calculations. ${ }^{36}$

Sequence and structure-based alignments. Representative sequences of each enzyme have been selected in $\mathrm{BLAST}^{37}$ and multiple-sequence alignments have been performed in ClustalOmega. ${ }^{38}$ Structure-based sequence alignments have been performed using T-COFFEE, ${ }^{39}$ as described previously. $^{40}$ 

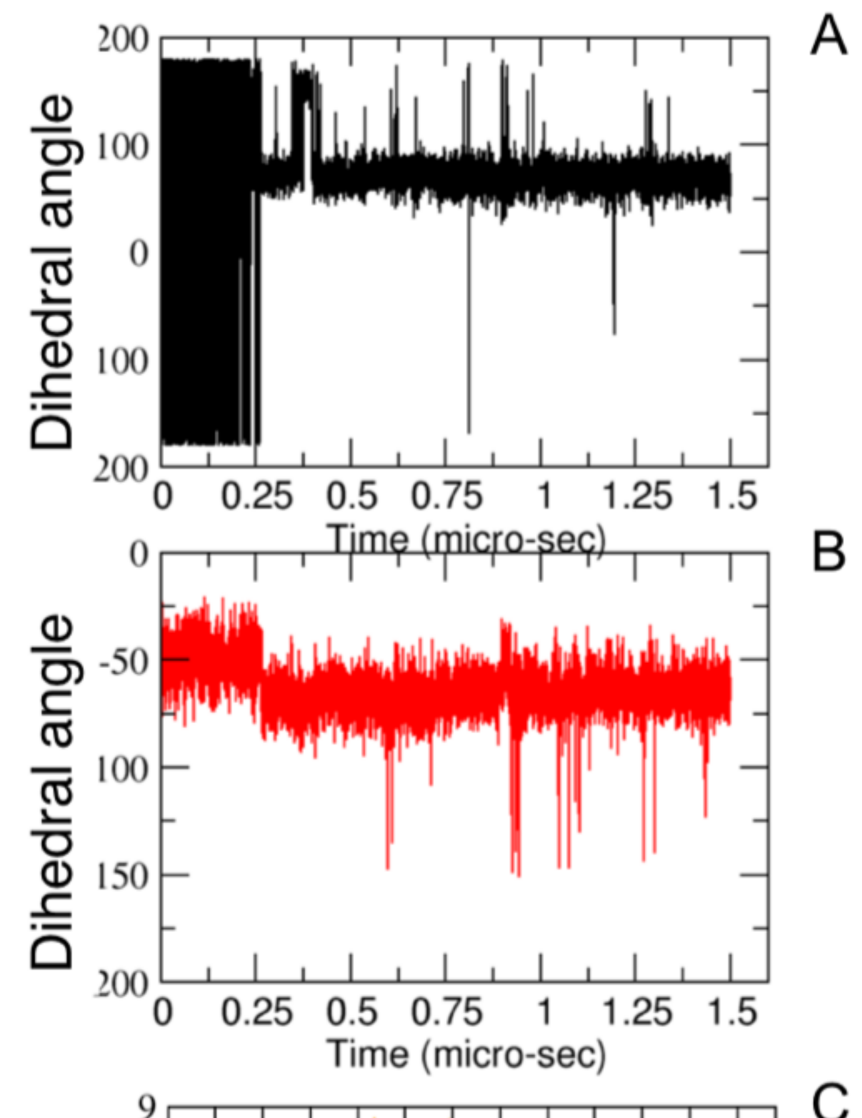

Supplementary Figure S1. Panel depicting time-dependent evolution of geometrical descriptors used to monitor active site rearrangement. Panel refers to one replica of a MD system where Lys131 is protonated. (A) $\psi$ dihedral angle formed by the atoms $\mathrm{C}-\mathrm{C} \alpha-\mathrm{C} \beta-\mathrm{C} \gamma$ of Lys131 that locates the residue position with respect to the Exo- $\lambda$ active core (see also Fig. 1 in the manuscript). (B) dihedral angle formed by the atoms $\mathrm{C} \alpha-\mathrm{C} \beta-\mathrm{C} \gamma-\mathrm{N}$ of Tyr154. (C) Evolution over the time of $d$-shuttle distance. 


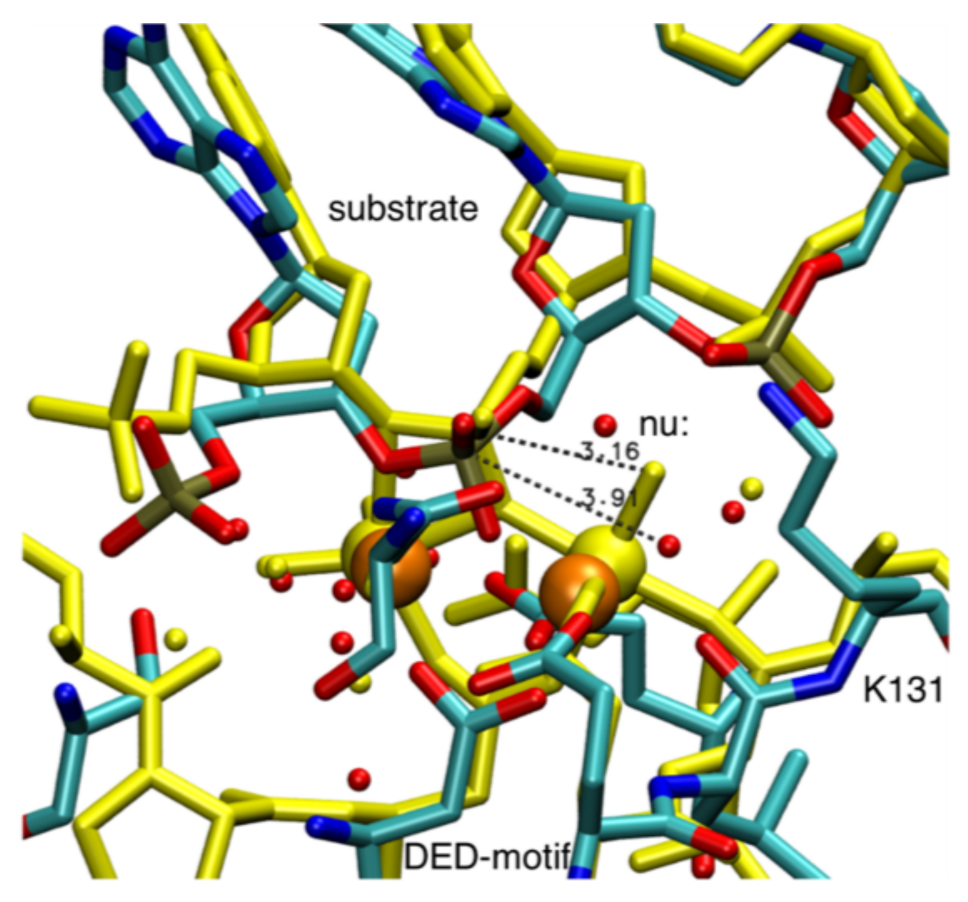

Supplementary Figure S2. Superimposition between the X-ray structure (PDBids 3SM4, in yellow) ${ }^{2}$ and a representative snapshot extracted from our MD simulations highlighting the active site of Exo$\lambda$. Orange spheres indicate catalytic metal ions while black dashed line indicates the length of $d$ newbond distance in the X-ray structure and from a snapshot extracted from our MD simulations. The picture highlights how the $d$-newbond length (i.e. nu-P distance) as well as the $\mathrm{Mg}$ geometry coordination are slightly distorted in presence of protonated Lys131. Hydrogen atoms were omitted for clarity. 


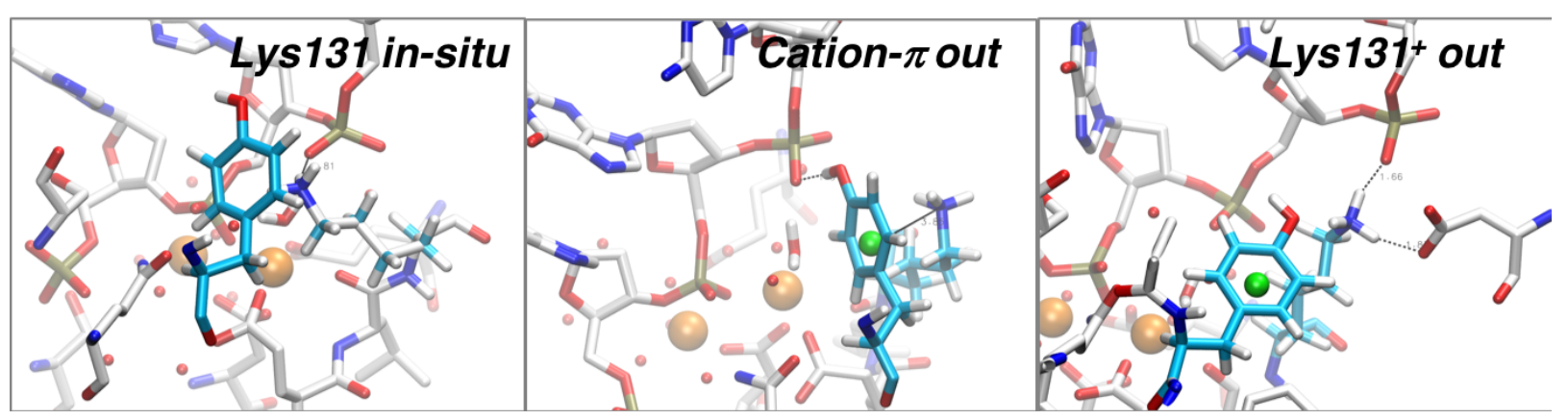

Supplementary Figure S3. Representative structures, extracted from MD simulations, highlighting the step-wise swing out process of the cation- $\pi$ interaction formed by Lys131 and Tyr154 in Exo- $\lambda$. Colour code: white carbons identify protein residues, while cyan carbons are those of amino acids involved in the formation of cation- $\pi$ interaction. Orange spheres represent the catalytically competent $\mathrm{Mg}^{2+}$ ions. The green sphere identifies the centre of mass of Tyr154 side-chain. Hydrogen atoms were omitted for clarity. 


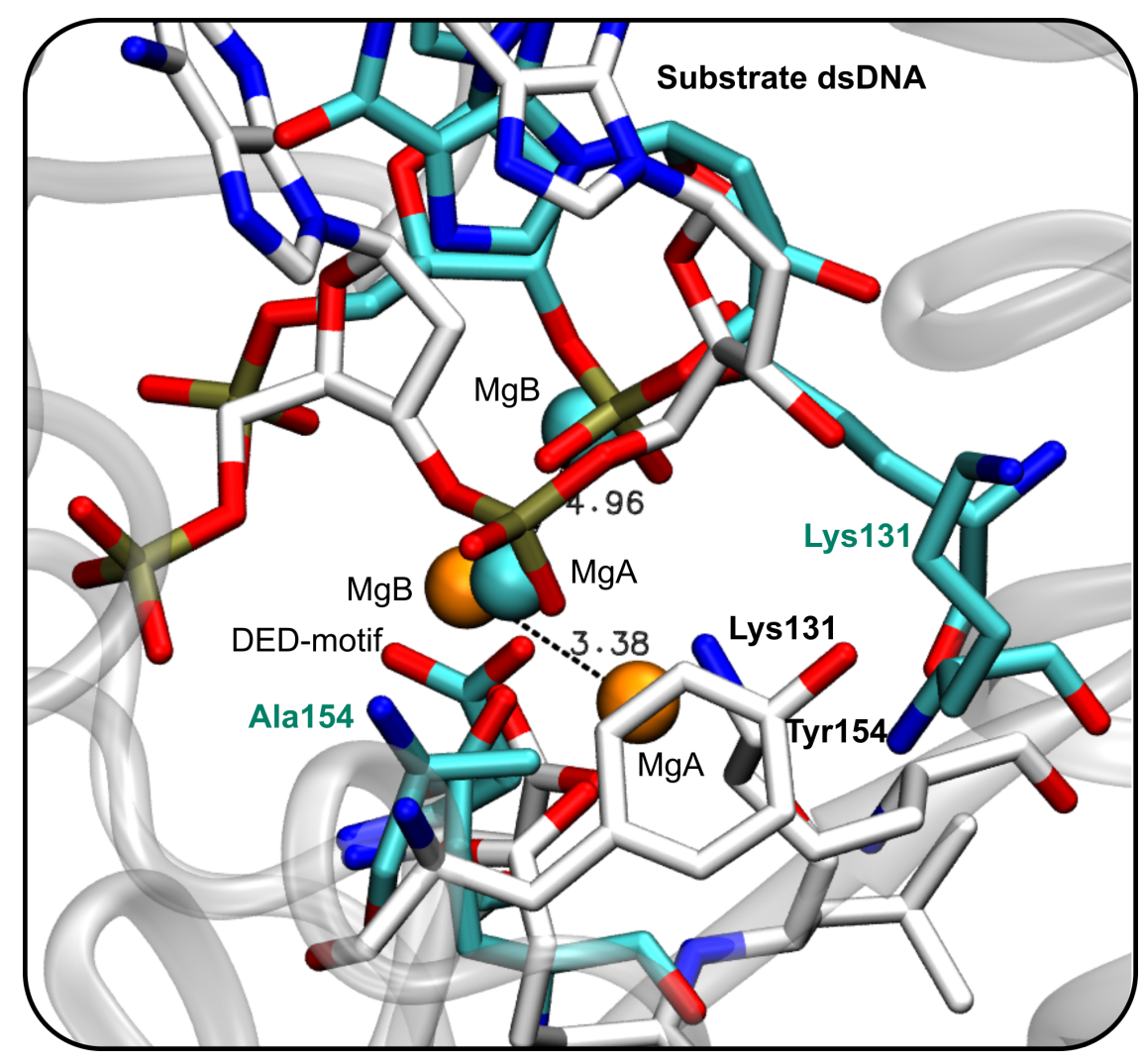

Supplementary Figure S4. Structural superimposition between a representative snapshot retrieved from our MD simulations (white backbone and orange magnesium ions for the wild-type Exo$\lambda /$ dsDNA binary complex) and Tyr154Ala mutant (cyan backbone and cyan magnesium ions). This structural superimposition shows the disruptive effect of Tyr154Ala mutation that determines the departure of Lys131 and a consequent relaxation of the local protein backbone (i.e. lack of cation- $\pi$ interaction). Besides this, the position of the scissile phosphate and location of the catalytic metal ions are also impacted. Indeed, the metal internuclear distance of $4.96 \AA$ does not fall in the optimum range compatible with a well-structured and prompt-to-react Michaelis-Menten complex. ${ }^{41-43}$ 


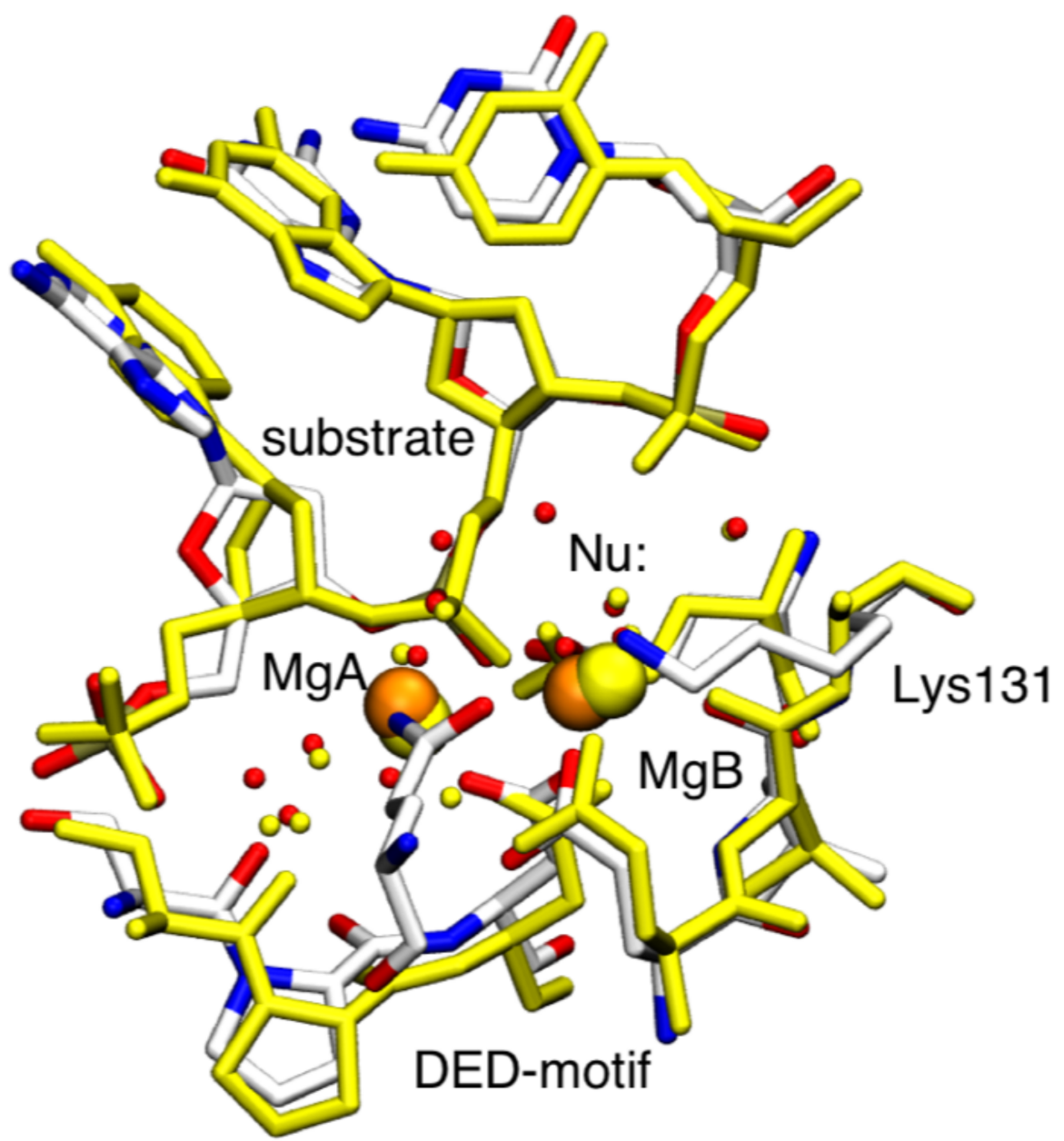

Supplementary Figure S5. Structural superimposition between a representative snapshot retrieved from our MD simulations (white backbone and orange magnesium ions) and X-ray structure (PDBid $3 \mathrm{SM} 4)^{2}$ of Exo- $\lambda$ active site. This structural superimposition shows the system in which Lys131 is deprotonated perfectly maintaining the active site geometry found in the crystal structure. Besides the position of the catalytic metal ions, also the nucleophilic water molecule (i.e. Nu:) overlaps well the one from the X-ray structure (highlighted in yellow). 


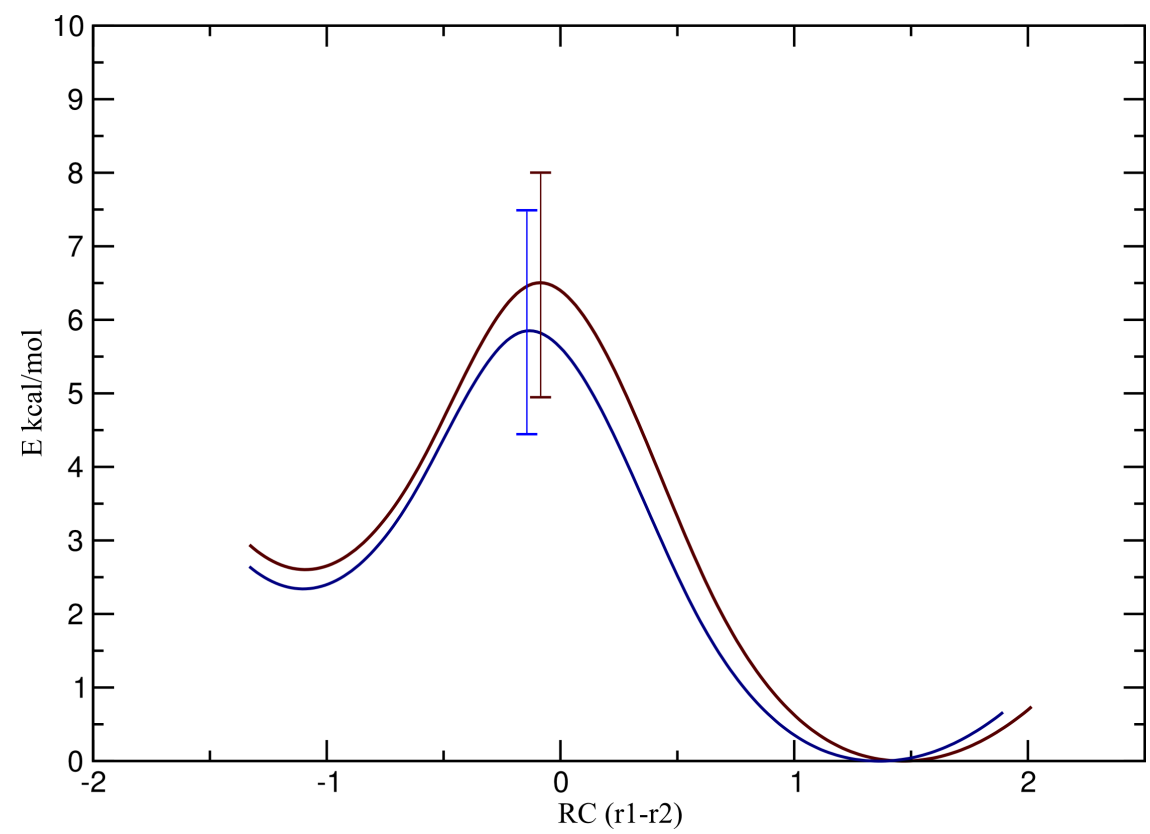

Supplementary Figure S6. Free-energy surface for the deprotonation of Lys131 through the conserved Asp138 (6.5 $\pm 1.6 \mathrm{kcal} / \mathrm{mol}$, black curve $)$ and via a bulk water molecule $(6.2 \pm 1.4 \mathrm{kcal} / \mathrm{mol}$, blue curve). See Figure 5 in the manuscript for structural details and Supplementary Text for the method used to reconstruct the free energy surfaces. 


\section{Legend:}

$>$ Escherichia_virus_lambda $>$ Enterobacteria phage_HK630 >Stx2-converting phage 1717 $>$ Achromobacter_sp_ATCC 35328 $>$ Cronobacter_phage_ENT47670 $>S$ pneumoniae

$>$ Vibrio phage 2E1

$>$ P multocida

$>$ H_parasuis

$>\mathrm{H}_{-}$influenzae

$>$ G_anatis

$>4$ WUZ:A| PDBID | CHAIN | SEQUENCE

>gi|428782822|ref|YP 007112573.1

>gi|209447136|ref|YP-002274221.1|

>gi|928599693| emb| CUKK07497.1|

>gi|431810511|ref|YP_007237585.1|

>gi|1062527211|ref|WP_069289300.1|

>gi| 1067529875 |gb|AOQ2 6699.1 |

>gi|1096225174|ref|WP 071171091.1|

$>$ gi|737515692|ref|WP_035494618.1|

>gi|491918549|ref|WP_005671505.1|

>gi|746077620|ref|WP 039145071.1

MTPDI I LQRTGI DVRAVEQGDDAWHKLRLGVITASEVHNVIAKPR---------------

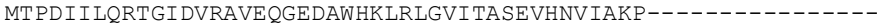

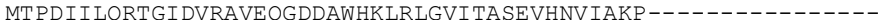

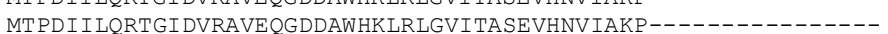

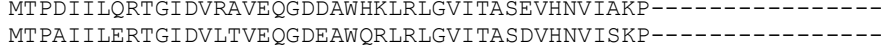

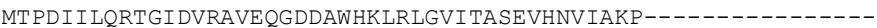
MI--NVNH ITGVNT FN IEQGTEEWLRHRAGTI TASRAHLVIADD IT PPMP DDVE I I PTEK

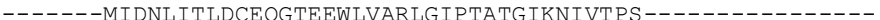
-------MIDGLITLDCEQGTEEWLVARLGIPTATGIKNIVNNS--------------$\star \star \star \star \star \star * \star *: \cdots$

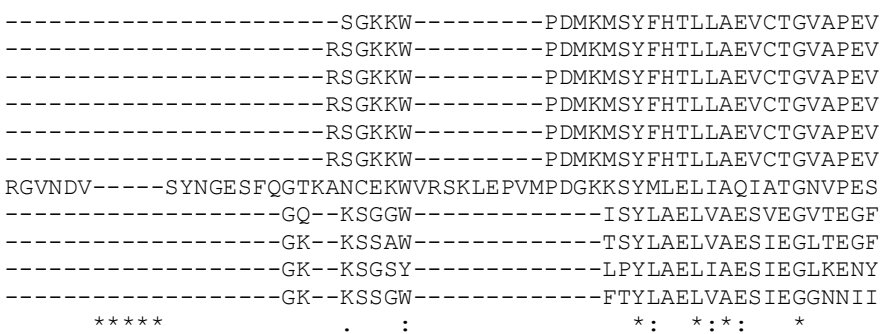
45 44 44 44 Enterobacteria_phāge_HK630 Stx2-converting phage 1717 Achromobacter sp ATCC $\overline{3} 5328$ Cronobacter_phage_ENT47670 S_pneumoniae Vibrio_phage_2E1

P multocida

H parasuis

$\mathrm{H}$ - influenzae

G_anatis

Escherichia virus lambda Enterobacteria_phāge_HK630 Stx2-converting_phagé_1717 Achromobacter_sp_ATCC 35328 Cronobacter_phage_ENT47670 S pneumoniae

Vibrio_phage_2E1

P_multocida

$\mathrm{H}$ parasuis

$\mathrm{H}$ influenzae

$\mathrm{G}^{-}$anatis

Escherichia virus lambda Enterobactéria phāge HK630 Stx2-converting phage 1717 Achromobacter_sp_ATCC $\overline{3} 5328$ Cronobacter_phage_ENT47670 S pneumoniae

Vibrio phage 2E1

P multocida

H_parasuis

H_influenzae

G_anatis

Escherichia_virus_lambda Enterobacteria_phage_HK630 Stx2-converting phage 1717 Achromobacter sp ATCC 35328 Cronobacter phage ENT47670 S_pneumoniae Vibrio_phage_2E1

P_multocida

H parasuis

$\mathrm{H}$ influenzae

G_anatis

NA-KALAWGKQYENDARTLFEFTSGVNVTESPIIYRDESMRTACSPDGLCS-DGNGLE I NA-KALAWGKQYENDARTLFEFTSGVNVTESP I IYRDESMRTACSPDGLCS-DGNGLEI NA-KALAWGKQYENDARTLFEFTSGVNVTESPIIYRDESMRTACSP DGLCS-DGNGLEL NA-KALAWGKQYENDARVLFEFTSGVNVTESPIIYRDESMRTACSPDGLCS-DGNGLEI NA-RALAWGKQYEDDARALFEFTAGVQVTESP I I YKDETMRTACSP DGLCS-DGRGLEI NA-KALAWGKOYENDARTLFEFTSGVNVTESPI I YRDE SMRTACS P DGLCS-DGNGLEI AS FKQAEWGHLNEPLARDAFEAKNFC IVTEAGL I YKDESLRCA I SP DGLLMDEKQGLE I KS-QHMERGNELEPLARMAYEFETGHDVTQVGGVYLNEKKELMVSPDGLI LSHQKGLE I KS-TDMLRGNLLEEQARMAYEFATGNDVVQVGGVYRNADKDMMVSPDGLI PTLRKGLE I KS-EDMARGNELEPFARAAYEFETGNAVIQVGGVYLNADKDLMISP DGLI PNLRKGLEI KT-VDMERGNELEPKARMAYEFLTDNTVVQVGGVYLNEQKELMISPDGLI PNLKKGLEIK 122

CPFTSRDFMKFRLGGFEAIKSAYMAQVQYSMWVTRKNAWYFANYDPRMK---REGLHYVV 188 CPFTSRDFMKFRLGGFEAIKSAYMAQVQYSMWVTRKNAWYFANYDPRMK---REGLHYVV 188 CPFTSRDFMKFRLGGFEAIKSAYMAQVQYSMWVTRKDAWYFANYDPRMK---REGLHYVV 188 CPFTSRDFMKFRLGGFEAIKSAYMAQVQYSMWVTRKDAWYFANYDPRMK---REGLHYVV 188 CPFTSRDFMKFRLGGFEAIKSAYMAQVQFSMWVTGKDAWYFSNYDPRMR---REGLHHVV 188 CPFTSRDFMKFRLGGFEAIKSAYMAQVQYSMWVTRKNAWYFANYDPRMK---REGLHYVV 188 SPYTTQVHLDTVLNG--KIKPEYLIQCQFSMWVTGWDKWHFCSYDHRLRGSSQNRLHTVV 231 CPKMKTHIKY ILEGG---VPSEYI IQVQVAMWVTGYKSWDFVSYCPEYQ---KQTLYLYT 176 CPKMKTHIKYIIEGV---VPSEYIIQVQVALWVTGYDSWDFVSYCPEYQ---KQTLFIHT 171 CPQIKTHIKYLLQGG---VPQEYLIQVQSALWVTGYETWDFVSYCPEYY---KQPFYLFT 176 CPKMKTHIKYLLESG---VPSEYLMQVQSALWVTGYETWDFVSYCPDYQ---KQPLYLYT 176

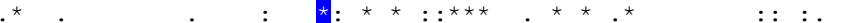

IERDEKYMASFDEIVPEFIEKMDEALAEIGFVFGEQWR-IERDEKYMASFDEIVPEFIEKMDEALAEIGFVFGEQWR-VERDEKYMASFDEMVPEFIEKMDEALAEIGFVFGEQWR-IERNEKYMASFDEMVPEF IEKMDEALAEIGFVFGEQWR-VERDEKYMEDFTEAVPEF IEKMDMALAE IGFTFGEQWR-IERDEKY

IERDES IMAKFDKY I PKF IDEMDRQLKKLNFE FNDQWREF ATRDEMLMKAFDEY IPQFLKSLR------ALRDG-----ENPDPVLMKAFDKY IPQFIETLK------ALKVN-----AQRDPNLMKSFDRLIPEFIKTLK------AYKSTE----AERDPI LMKAFDKY I PEFLNALK------ALKGENQWQA- 


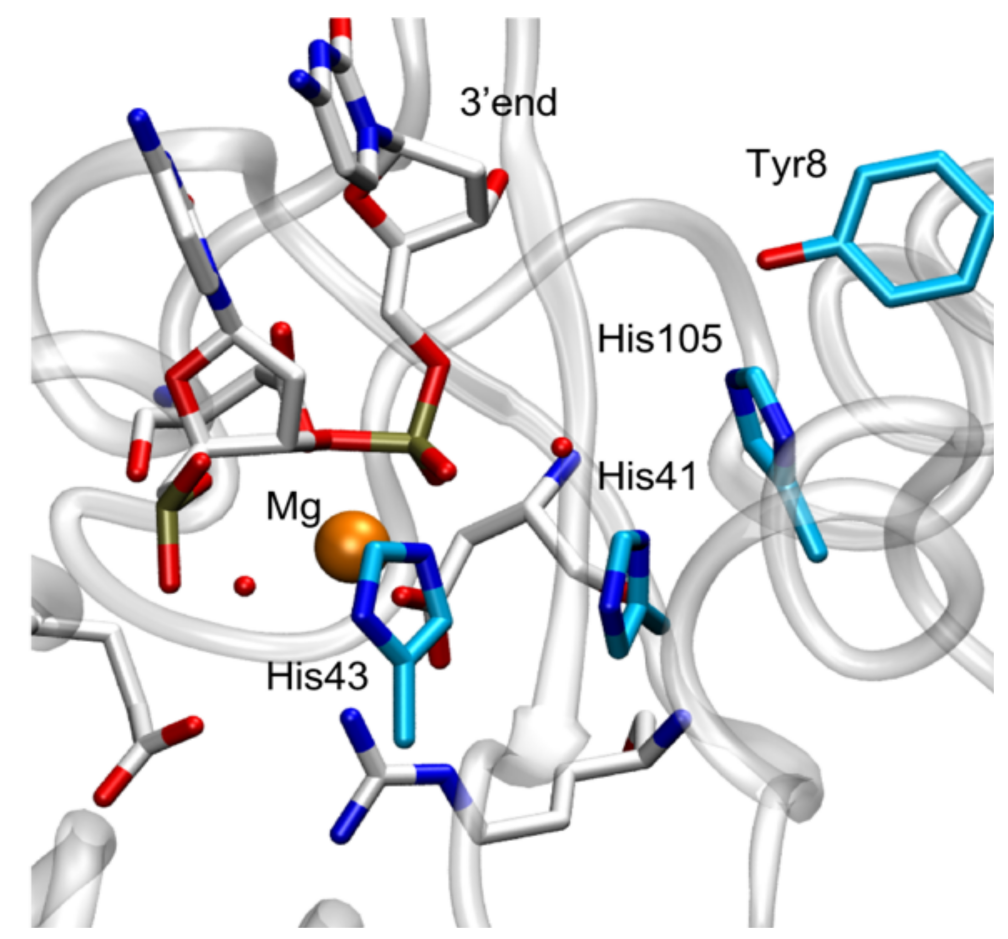

Supplementary Figure S8. Close-view of the active site of phage T4 endonuclease VII (PDBid 2QNC). ${ }^{44}$ The histidine triad is depicted with carbon in cyan. Tyr8 might alleviate the positive charge build up after proton-abstraction from the Mg-oriented water molecule (red dot). Substrate is reported with white carbons. 


\section{References}

(1) Zhang, J.; Pan, X.; Bell, C. E.; Crystal structure of lambda exonuclease in complex with DNA and $\mathrm{Ca}(2+)$. Biochemistry. 2014, 53, 7415-7425.

(2) Zhang, J.; McCabe, K. A.; Bell, C. E.; Crystal structures of lambda exonuclease in complex with DNA suggest an electrostatic ratchet mechanism for processivity. Proc. Natl. Acad. Sci. U.S.A. 2011, 108, 11872-11877.

(3) Maier, J. A.; Martinez, C.; Kasavajhala, K.; Wickstrom, L.; Hauser, K. E.; Simmerling, C.; ff14SB: Improving the Accuracy of Protein Side Chain and Backbone Parameters from ff99SB. $J$ Chem. Theory. Comput. 2015, 11, 3696-3713.

(4) Ivani, I.; Dans, P. D.; Noy, A.; Perez, A.; Faustino, I.; Hospital, A.; Walther, J.; Andrio, P.; Goni, R.; Balaceanu, A.; Portella, G.; Battistini, F.; Gelpi, J. L.; Gonzalez, C.; Vendruscolo, M.; Laughton, C. A.; Harris, S. A.; Case, D. A.; Orozco, M.; Parmbsc1: a refined force field for DNA simulations. Nat. Methods. 2016, 13, 55-58.

(5) Hess, B.; Bekker, H.; Berendsen, H. J. C.; Fraaije, J. G. E. M.; LINCS: A linear constraint solver for molecular simulations. J. Comp. Chem. 1997, 18, 1463-1472.

(6) Berendsen, H. J. C.; Vanderspoel, D.; Vandrunen, R.; Gromacs - a Message-Passing Parallel Molecular-Dynamics Implementation. Comput. Phys. Commun. 1995, 91, 43-56.

(7) Grest, G. S.; Kremer, K.; Molecular-Dynamics Simulation for Polymers in the Presence of a Heat Bath. Phys. Rev. A. 1986, 33, 3628-3631.

(8) Feller, S. E.; Zhang, Y. H.; Pastor, R. W.; Brooks, B. R.; Constant-Pressure MolecularDynamics Simulation - the Langevin Piston Method. J. Chem. Phys. 1995, 103, 4613-4621.

(9) Jorgensen, W. L.; Chandrasekhar, J.; Madura, J. D.; Impey, R. W.; Klein, M. L.; Comparison of Simple Potential Functions for Simulating Liquid Water. J. Chem. Phys. 1983, 79, 926-935.

(10) Car, R.; Parrinello, M.; Unified Approach for Molecular-Dynamics and Density-Functional Theory. Phys. Rev. Lett. 1985, 55, 2471-2474.

(11) Sham, W. K. a. L. J.; Self-Consistent Equations Including Exchange and Correlation Effects. Phys. Rev. Lett. 1965, 140.

(12) Laio, A.; VandeVondele, J.; Rothlisberger, U.; A Hamiltonian electrostatic coupling scheme for hybrid Car-Parrinello molecular dynamics simulations. J. Chem. Phys. 2002, 116, 6941-6947.

(13) Becke, A. D.; Density-Functional Exchange-Energy Approximation with Correct Asymptotic-Behavior. Phys. Rev. A. 1988, 38, 3098-3100.

(14) Lee, C. T.; Yang, W. T.; Parr, R. G.; Development of the Colle-Salvetti Correlation-Energy Formula into a Functional of the Electron-Density. Phys. Rev. B. 1988, 37, 785-789. 
(15) De Vivo, M.; Dal Peraro, M.; Klein, M. L.; Phosphodiester cleavage in ribonuclease H occurs via an associative two-metal-aided catalytic mechanism. J. Am. Chem. Soc. 2008, 130, 10955-10962. (16) Dal Peraro, M.; Ruggerone, P.; Raugei, S.; Gervasio, F. L.; Carloni, P.; Investigating biological systems using first principles Car-Parrinello molecular dynamics simulations. Curr. Opin. Struct. Biol. 2007, 17, 149-156.

(17) Iannuzzi, M.; Laio, A.; Parrinello, M.; Efficient exploration of reactive potential energy surfaces using Car-Parrinello molecular dynamics. Phys. Rev. Lett. 2003, 90, 238302.

(18) De Vivo, M.; Ensing, B.; Klein, M. L.; Computational study of phosphatase activity in soluble epoxide hydrolase: high efficiency through a water bridge mediated proton shuttle. J. Am. Chem. Soc. 2005, 127, 11226-11227.

(19) De Vivo, M.; Bridging quantum mechanics and structure-based drug design. Front. Biosci. (Landmark Ed). 2011, 16, 1619-1633.

(20) De Vivo, M.; Ensing, B.; Dal Peraro, M.; Gomez, G. A.; Christianson, D. W.; Klein, M. L.; Proton shuttles and phosphatase activity in soluble epoxide hydrolase. J. Am. Chem. Soc. 2007, 129, 387-394.

(21) Troullier, N.; Martins, J. L.; Efficient pseudopotentials for plane-wave calculations. Phys. Rev. B. Cond. Matt. 1991, 43, 1993-2006.

(22) von Lilienfeld, O. A.; Tavernelli, I.; Rothlisberger, U.; Sebastiani, D.; Variational optimization of effective atom centered potentials for molecular properties. J. Chem. Phys. 2005, 122, 14113.

(23) Martyna, G. J.; A reciprocal space based method for treating long range interactions in ab initio and force-field-based calculations in clusters. J. Chem. Phys. 1999, 110, 2810-2821.

(24) De Vivo, M.; Cavalli, A.; Carloni, P.; Recanatini, M.; Computational study of the phosphoryl transfer catalyzed by a cyclin-dependent kinase. Chemistry. 2007, 13, 8437-8444.

(25) Cavalli, A.; De Vivo, M.; Recanatini, M.; Density functional study of the enzymatic reaction catalyzed by a cyclin-dependent kinase. Chem. Commun. 2003, 1308-1309.

(26) W. F. van Gunsteren, S. R. B., A. A. Eising, P. H. Hünenberger, P. Krüger, A. E. Mark, W. R. P. Scott, and I. G. Tironi; Biomolecular Simulation: The GROMOS96 Manual and User Guide. Vdf Hochschulverlag AG an der ETH Zürich. 1996, 1-1042.

(27) Mendieta-Moreno, J. I.; Walker, R. C.; Lewis, J. P.; Gomez-Puertas, P.; Mendieta, J.; Ortega, J.; FIREBALL/AMBER: An Efficient Local-Orbital DFT QM/MM Method for Biomolecular Systems. J. Chem. Theory Comput. 2014, 10, 2185-2193. 
(28) Case, D. A.; Cheatham, T. E., 3rd; Darden, T.; Gohlke, H.; Luo, R.; Merz, K. M., Jr.; Onufriev, A.; Simmerling, C.; Wang, B.; Woods, R. J.; The Amber biomolecular simulation programs. J. Comput. Chem. 2005, 26, 1668-1688.

(29) Ufimtsev, I. S.; Martinez, T. J.; Quantum Chemistry on Graphical Processing Units. 3. Analytical Energy Gradients, Geometry Optimization, and First Principles Molecular Dynamics. $J$. Chem. Theory Comput. 2009, 5, 2619-2628.

(30) Gotz, A. W.; Clark, M. A.; Walker, R. C.; An extensible interface for QM/MM molecular dynamics simulations with AMBER. J. Comput. Chem. 2014, 35, 95-108.

(31) Kästner, J.; Umbrella Sampling. WIREs Comput. Mol. Sci. 2011, 1, 10.

(32) Torrie, G. M. V., J. P.; Nonphysical sampling distributions in Monte Carlo free-energy estimation: Umbrella sampling. J. Comp. Phys. 1977, 23, 12.

(33) Dreyer, J. B., G.; Ippoliti, E.; Genna, V.; De Vivo, M.; Carloni, P.; Rothlisberger, U. In Simulating Enzyme Reactivity: Computational Methods in Enzyme Catalysis; Tuñon, I., Moliner, V., Eds.; Royal Society of Chemistry: London, 2016; p 246.

(34) Kumar, S. R., J. M.; Bouzida, D.; Swendsen, R. H.; Kollman, P. A.; Multidimensional freeenergy calculations using the weighted histogram analysis method. J. Comput. Chem. 1995, 16, 11.

(35) Hub, J. S.; de Groot., B. L; Van Der Spoel, D.; G-whams-a free Weighted Histogram Analysis implementation including robust error and autocorrelation estimates. J. Chem. Theory Comput. 2010, 6, 3713-3720.

(36) Fox, S. J.; Pittock, C.; Fox, T.; Tautermann, C. S.; Malcolm, N.; Skylaris, C. K.; Electrostatic embedding in large-scale first principles quantum mechanical calculations on biomolecules. J. Chem. Phys. 2011, 135, 224107.

(37) Altschul, S. F.; Gish, W.; Miller, W.; Myers, E. W.; Lipman, D. J.; Basic local alignment search tool. J Mol Biol. 1990, 215, 403-410.

(38) Sievers, F.; Wilm, A.; Dineen, D.; Gibson, T. J.; Karplus, K.; Li, W.; Lopez, R.; McWilliam, H.; Remmert, M.; Soding, J.; Thompson, J. D.; Higgins, D. G.; Fast, scalable generation of highquality protein multiple sequence alignments using Clustal Omega. Mol. Sys. Biol. 2011, 7, 539.

(39) Notredame, C.; Higgins, D. G.; Heringa, J.; T-Coffee: A novel method for fast and accurate multiple sequence alignment. J. Mol. Biol. 2000, 302, 205-217.

(40) Marcia, M.; Ermler, U.; Peng, G.; Michel, H.; A new structure-based classification of sulfide:quinone oxidoreductases. Proteins. 2010, 78, 1073-1083.

(41) Genna, V.; Colombo, M.; De Vivo, M.; Marcia, M.; Second-Shell Basic Residues Expand the Two-Metal-Ion Architecture of DNA and RNA Processing Enzymes. Structure. 2018, 26, 40-50. 
(42) Genna, V.; Donati, E.; De Vivo, M. ; The Catalytic Mechanism of DNA and RNA Polymerases. ACS Catal. 2018, 8, 11103-11118.

(43) Palermo, G.; Cavalli, A.; Klein, M. L.; Alfonso-Prieto, M.; Dal Peraro, M.; De Vivo, M.; Catalytic metal ions and enzymatic processing of DNA and RNA. Acc. Chem. Res. 2015, 48, 220228.

(44) Biertumpfel, C.; Yang, W.; Suck, D.; Crystal structure of T4 endonuclease VII resolving a Holliday junction. Nature. 2007, 449, 616-620. 